\title{
Synergistic Effect of P[MPEGMA-IL] Modified Graphene on Morphology and Dielectric Properties of PLA/PCL Blends
}

\author{
Yiyang Zhou, ${ }^{1}$ Ping Wang, ${ }^{2}$ Gang Ruan, ${ }^{1}$ Pei $\mathrm{Xu}^{1, *}$ and Yunsheng Ding ${ }^{1, *}$
}

\begin{abstract}
To investigate the combined effect of graphene (Gra) and poly(methoxy poly(ethylene glycol) monomethacrylate-co-1-vinyl3-ethylimidazolium bromide) (P[MPEGMA-IL]) on the morphology, crystallization behavior and dielectric properties for polylactide (PLA)/poly( $\varepsilon$-caprolactone) (PCL) blends, a series of PLA/PCL blend based composites have been prepared using solution-cast method. The P[MPEGMA-IL] modified Gra could be mainly localized in the PCL domains, and improved the dispersibility of PCL phase in the composites. Due to the heterogeneous nucleation of Gra and ion cluster of P[MPEGMA-IL], the crystallinity of PLA and PCL were improved simultaneously with the increase of modified Gra content. The good conductivity of Gra and the better dispersion of PCL made the PLA/PCL blends with higher dielectric permittivity owing to the increase of the mobile charge carriers at the interfaces.
\end{abstract}

Keywords: Polymeric composites; Graphene; Ionic liquid; Interfaces; Dielectric properties.

Received: 8 September 2020; Accepted date: 27 October 2020.

Article type: Research article.

\section{Introduction}

With the increasing shortage of oil resources and the serious environmental problem, polylactide (PLA) as a biodegradable thermoplastic polymer has received much attention in recent years. ${ }^{[1-3]}$ It has good application prospects in electronic fields because of easy processability and good mechanical strength. However, poor tensile toughness and low impact strength limit its applications. ${ }^{[4,5]}$ The mechanical properties and crystallinity of PLA can be greatly improved by adding some plasticizers, nanofillers, or blending with other rubbery biodegradable polymers. ${ }^{[6-9]}$ The rubbery and biodegradable poly( $\varepsilon$-caprolactone) (PCL) has good toughness and its ductility even exceeds the low density polyethylene used as a film material, which can effectively improve the problem of the brittleness of PLA. ${ }^{[10-12]}$ The common compatibilizing agents such as PLA-PCL random, PLA-PCL-PLA block copolymers can improve the interfacial adhesion and enhance the miscibility of the two

\footnotetext{
${ }^{1}$ Department of Polymer Science and Engineering, School of Chemistry and Chemical Engineering, and Anhui Key Laboratory of Advanced Functional Materials and Devices, Hefei University of Technology, Hefei 230009, China.

${ }^{2}$ School of Materials and Chemical Engineering, Anhui Jianzhu University, Hefei 230601, China.

*E-mail: chxuper@hfut.edu.cn (P. Xu), dingys@hfut.edu.cn (Y. Ding).
}

components. ${ }^{[13-15]}$

Graphene (Gra) is a two-dimensional carbon material with a single atomic layer ${ }^{[16,17]}$ The tight bonding of Gra surface and the delocalized electrons generated by the $\mathrm{sp}^{2}$ hybridization of the carbon atoms give excellent mechanical property, charge mobility and electrical conductivity. ${ }^{[18-20]}$ Gra can be used as conductive filler to improve dielectric constant of polymer composites near percolation threshold. ${ }^{[21,22]}$ Ionic liquids are usually composed of relatively large, asymmetrical organic cations and inorganic anions of relatively small size. ${ }^{[23]}$ The compatibility of the biodegradable PLA/PCL blend was improved in the presence of organoclays and organoclay/graphene mixtures. ${ }^{[24]}$ The use of filler mixtures to prepare composite materials is a very interesting way to have the best properties using classical methods of preparation.

Ionic liquids are known for their good thermal stability, chemical stability, electrical conductivity and low vapor pressure. ${ }^{[26,27]}$ Ionic liquid-containing diblock copolymer can be used as compatibilizer to control interfacial structure and corresponding properties of incompatible bio-polyester blends. ${ }^{[28]}$ There was a non-covalent interaction between ionic liquid and Gra, which promoted the dispersion of Gra. ${ }^{[29,30]}$ The fillers such as Gra, CNTs and ionic liquid could selectively localized at the PCL phase and phase interface, and the change of viscosity ratio of PLA/PCL induce phase inversion of PLA/PCL blends. ${ }^{[28,31-32]}$ 
In this work, poly (methoxy poly (ethylene glycol) monomethacrylate-co-1-vinyl-3-ethy limidazolium bromide (P[MPEGMA-IL]) and Gra were used to control the morphological structure of PLA/PCL blends and the dielectric properties of the blends. The objective of this study is to explore the effect of distribution and dispersion of Gra modified by P[MPEGMA-IL] on the phase morphology and the dielectric properties of the blends.

\section{Experimental}

\subsection{Materials}

Polylactide (PLA, grade 4032D) was purchased from Nature works Co., Ltd. Polycaprolactone (PCL) was purchased from Solvay Co., Ltd. Graphene (Gra, 0.5-2 $\mu \mathrm{m}$ in diameter, 0.8$1.2 \mathrm{~nm}$ thickness) was obtained from Nanjing XFNANO Materials Tech Co., Ltd. 1-vinyl-3-ethylimidazolium bromide ionic liquid (IL) was obtained from Lanzhou Institute of Chemical Physics, China. Methoxy poly (ethyleneglycol) monomethacrylate (MPEGMA) was purchased from Aladdin Chemical Co. The synthesis and characterization of P[MPEGMA-IL] copolymer were displayed in Fig. S1.

\subsection{Sample preparation}

The PLA/PCL/P[MPEGMA-IL]/Gra composites were prepared by solution mixing. Firstly, the P[MPEGMA-IL] were dissolved in dichloromethane (DCM). Then Gra was added to the solution with the aid of sonication for $2 \mathrm{~h}$. PLA and PCL were also dissolved in DCM, and the mass ratio of between PLA and PCL was 7:3. The P[MPEGMA-IL]/Gra dispersion liquid was further mixed with polymer solution at $40{ }^{\circ} \mathrm{C}$ by using magnetic stirring and sonication. Until the solvent volatilized completely, the samples were dried at $40{ }^{\circ} \mathrm{C}$ for $4 \mathrm{~h}$ in oven and were then dried at $40{ }^{\circ} \mathrm{C}$ for $24 \mathrm{~h}$ in a vacuum oven. The samples were hot pressed into diskshaped films with the thickness all around $100 \mu \mathrm{m}$ at $10 \mathrm{MPa}$ and $185{ }^{\circ} \mathrm{C}$. The component mass ratio of PLA/PCL/P[MPEGMA-IL]/Gra blends was shown in Table 1.

\subsection{Characterization}

The thermal properties of the composites were investigated using a differential scanning calorimetry (DSC) (model Q2000, TA Instruments). The samples were held at $190{ }^{\circ} \mathrm{C}$ for 5 minutes to eliminate the thermal history. All samples were cooled down to $-40{ }^{\circ} \mathrm{C}$ at the cooling rate of $10^{\circ} \mathrm{C} / \mathrm{min}$, and finally heated to $250{ }^{\circ} \mathrm{C}$ at the same heating rate. The degree of crystallinity, $X_{\mathrm{C}}$, of samples was calculated by the following equation:

$$
X_{\mathrm{C}}=\frac{\Delta H_{\mathrm{m}}-\Delta H_{\mathrm{cc}}}{w \Delta H_{\mathrm{m}}^{\mathrm{o}}} \times 100 \%
$$

where $\Delta H_{\mathrm{m}}$ and $\Delta H_{\mathrm{cc}}$ are the measured enthalpies of melting and cold crystallization for the homopolymer of PLA or PCL in the blends, and $w$ is the weight fraction of PLA or PCL in the blends. $\Delta H_{\mathrm{m}}^{\mathrm{o}}$ is the melting enthalpy of $100 \%$ crystalline polymer $(93.6 \mathrm{~J} / \mathrm{g}$ for PLA, $136.0 \mathrm{~J} / \mathrm{g}$ for PCL, respectively). ${ }^{[13,14]}$ The morphologies of the samples were investigated using a JSM-6490LV scanning electron microscope (SEM). The samples were first freeze-fractured in liquid nitrogen, and then the PCL minor phase was etched with acetic acid for $15 \mathrm{~h}$ at room temperature. The etched fractured surface was sputter-coated with metal. The dielectric properties were measured using an Agilent E4980A Impedance Analyzer (USA). Both sides of the samples were coated of $10 \mathrm{~mm}$ diameter with silver as electrodes.

\section{Results and discussion \\ 3.1 Morphologies of the PLA/PCL/Gra/P[MPEGMA-IL] composites}

Fig. 1 shows the SEM images of the PLA/PCL blends filled with Gra after glacial acetic acid etching. There were voids of different sizes after the PCL phase was etched. As seen in Fig. 1, the shape of PCL particles was irregular in the PLA/PCL blend system, corresponding to the typical structure of immiscible blends. The incorporation of low content of Gra improved the compatibilization between PLA and PCL and decreases the agglomeration of PCL, but when the Gra content was increased, the dispersion of PCL phase became worse. The reason for the phenomenon is that Gra can increase the viscosity of PCL phase, cause smaller spherical nodules of PCL phase, and promote the dispersion of PCL ${ }^{[33]}$ But Gra tended to aggregate easily, and the size of PCL phase became larger in the PLA/PCL/0.75Gra composites.

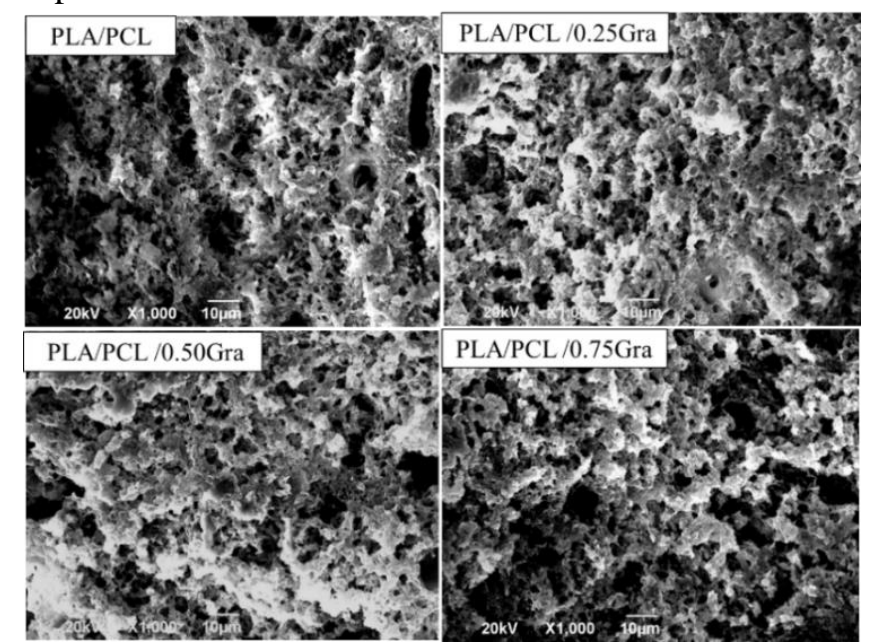

Fig. 1 The SEM images of PLA/PCL blends filled with Gra.

The SEM images of the PLA/PCL blends filled with Gra and P[MPEGMA-IL] after glacial acetic acid etching are shown in Fig. 2. Compared to PLA/PCL blend and PLA/PCL/0.25Gra composites, the particle size distributions of PCL droplets were more uniform in the PLA/PCL blend containing 4P[MPEGMA-IL]. The main reason for this phenomenon is that the MEPGMA groups of P[MEPGMAIL] have a high affinity for both polymer phases, and the low viscosity of PCL phase is easy to diffuse P[MEPGMA-IL]. Hence, after adding 4P[MPEGMA-IL], P[MEPGMA-IL] could be mainly localized in the PCL domains, and the dispersion of PCL was improved. The PCL phase size of PLA/PCL/8P[MPEGMA-IL] blend was significantly larger than that of PLA/PCL/4P[MPEGMA-IL] blend. A portion of 
Table 1 The component of PLA/PCL/P[MPEGMA-IL]/Gra composites.

\begin{tabular}{ccccc}
\hline \multirow{2}{*}{ Sample } & \multicolumn{2}{c}{ Matrix (wt\%) } & \multicolumn{2}{c}{ Filler (phr) } \\
\cline { 2 - 5 } & PLA & PCL & P[MPEGMA-IL] & Gra \\
\hline PLA/PCL & 70 & 30 & 0 & 0 \\
PLA/PCL/0.25Gra & 70 & 30 & 0 & 0.25 \\
PLA/PCL/0.50Gra & 70 & 30 & 0 & 0.50 \\
PLA/PCL/0.75Gra & 70 & 30 & 0 & 0.75 \\
PLA/PCL/4P[MPEGMA-IL] & 70 & 30 & 4 & 0 \\
PLA/PCL/8P[MPEGMA-IL] & 70 & 30 & 8 & 0 \\
PLA/PCL/0.25Gra/4P[MPEGMA-IL] & 70 & 30 & 4 & 0.25 \\
PLA/PCL/0.25Gra/8P[MPEGMA-IL] & 70 & 30 & 8 & 0.25 \\
PLA/PCL/0.50Gra/8P[MPEGMA-IL] & 70 & 30 & 8 & 0.50 \\
PLA/PCL/0.75Gra/8P[MPEGMA-IL] & 70 & 30 & 8 & 0.75 \\
\hline
\end{tabular}

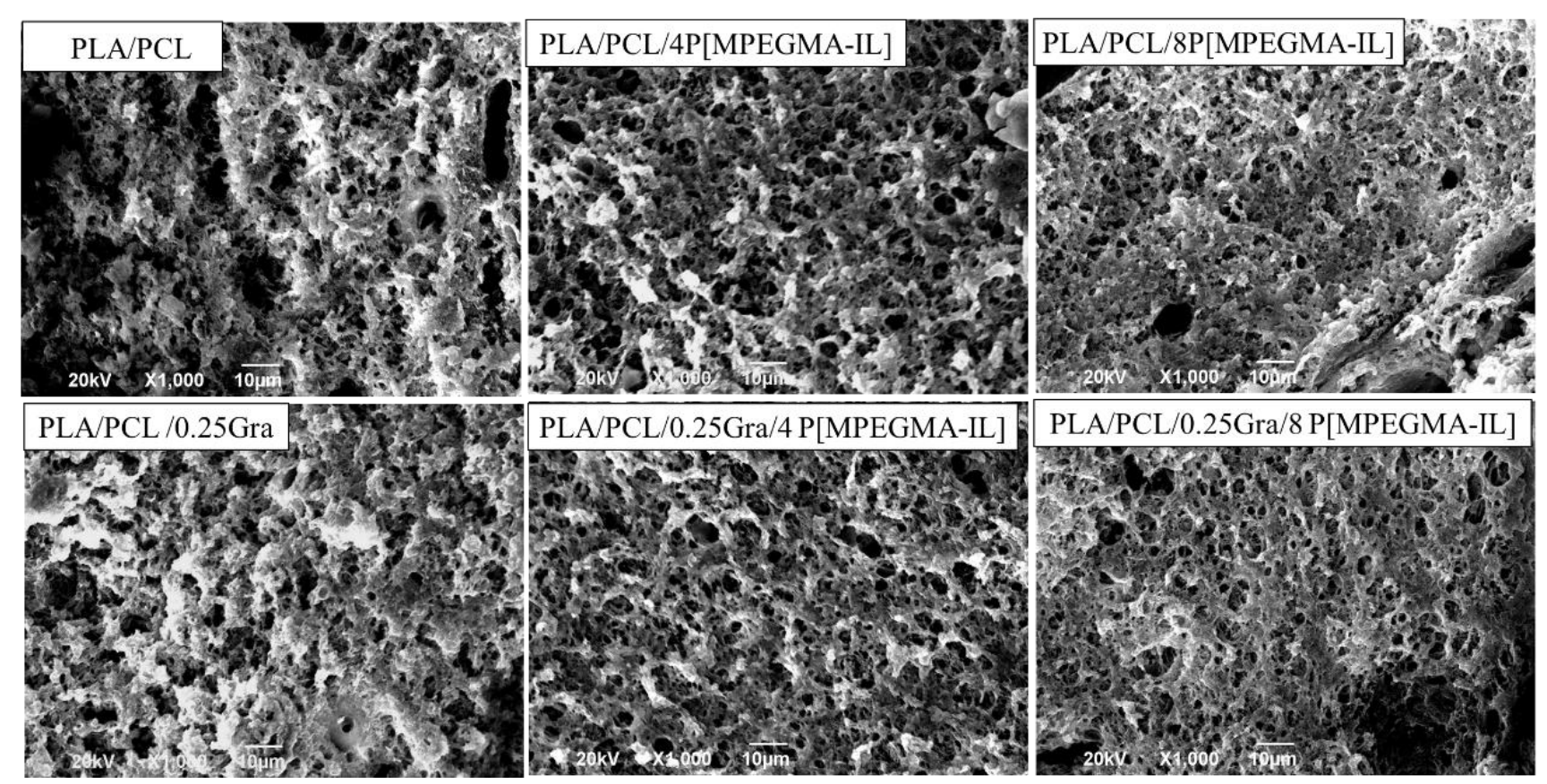

Fig. 2 The SEM micrographs of PLA/PCL blends filled with Gra and P[MPEGMA-IL].
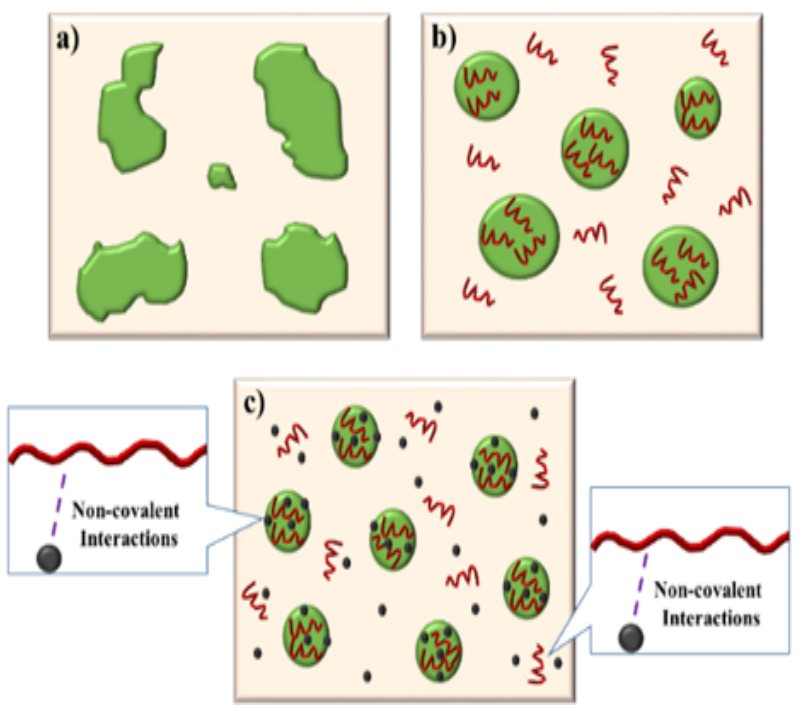

Fig. 3 Schematic illustration of the morphology of (a) PLA/PCL blend, (b) PLA/PCL/P[MPEGMA-IL] blends, and (c) PLA/PCL/Gra/P[MPEGMA-IL] composites. 

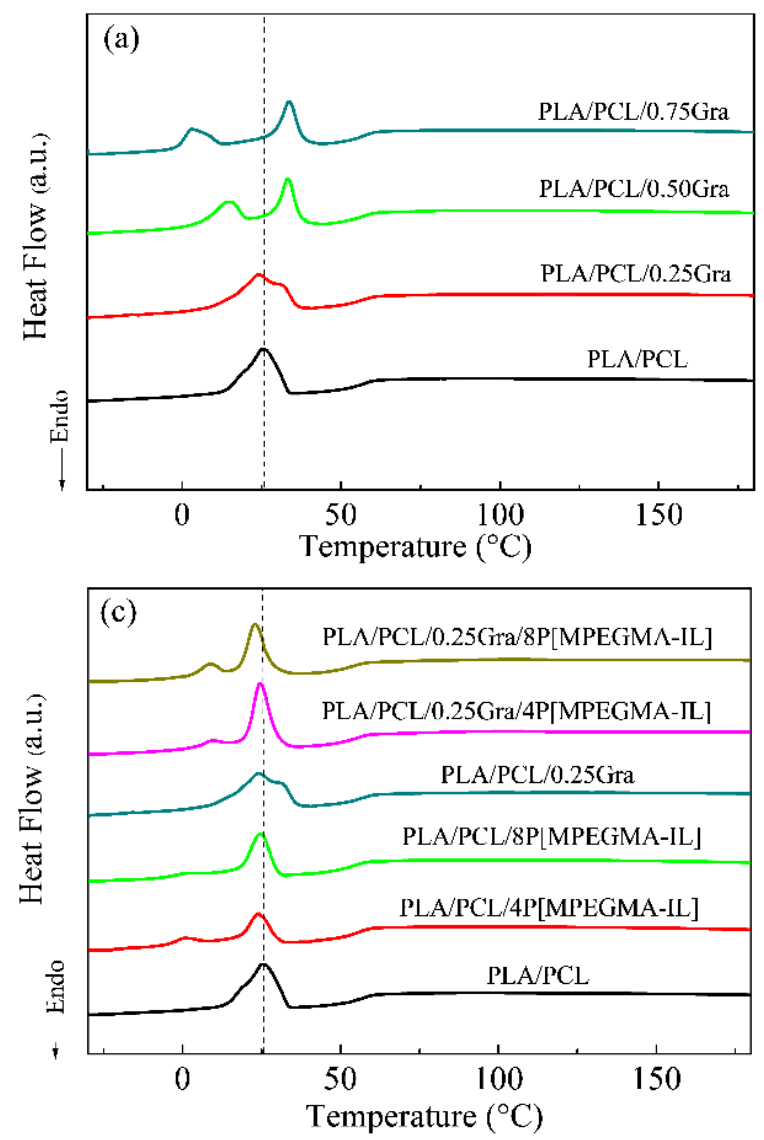
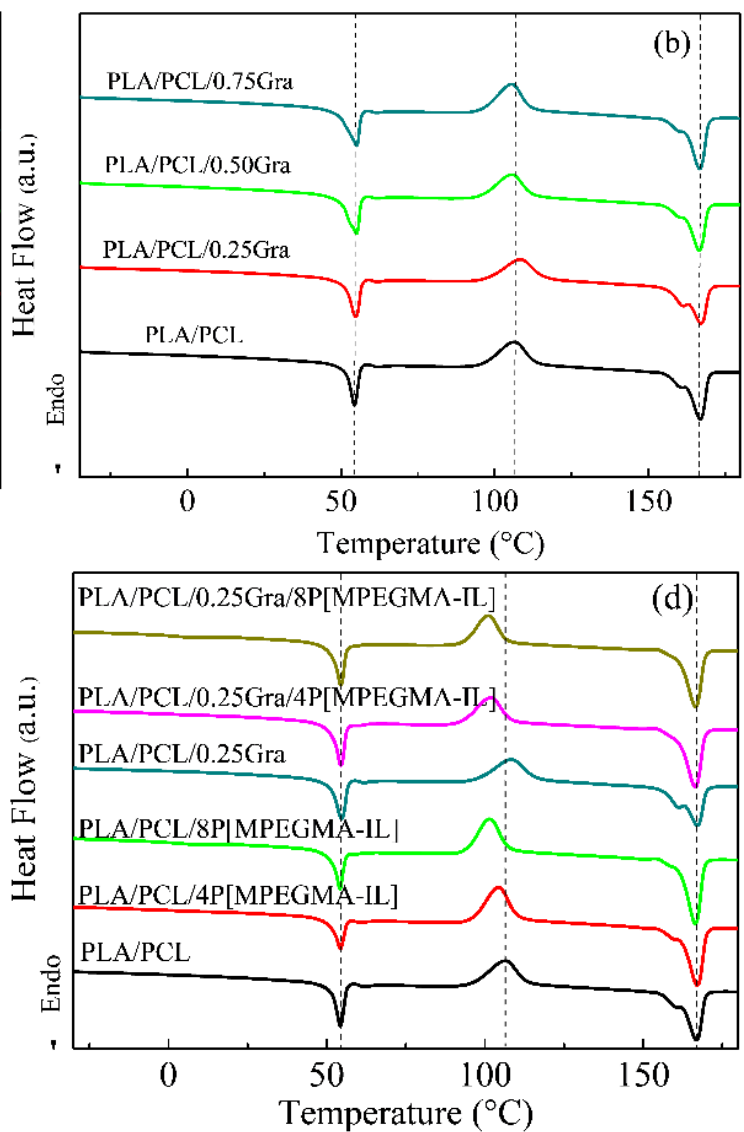

Fig. 4 DSC cooling curves of (a) PLA/PCL/Gra and (b) heating curves of PLA/PCL/Gra; (c) cooling curves of PLA/PCL/Gra/P[MPEGMA-IL] and (d) heating curves of PLA/PCL/Gra/P[MPEGMA-IL].

8P[MPEGMA-IL] distributed in the PLA phase was increased, further changing the viscosity of the PLA phase, and making partial aggregation of PCL phase. ${ }^{[11]}$ But after 8P[MPEGMA-IL] blends, PCL had the best dispersion in the PLA matrix. Gra could be non-covalently modified by P[MEPGMA-IL] via the short-ranged cation- $\pi$ interactions. The Gra tended to be dispersed in the PCL phase owing to the interaction force and the viscosity change of PCL with the introduction of P[MPEGMA-IL]. Moreover, P[MPEGMA-IL] could also improve the dispersion of Gra in the polymer matrix, so the dispersion of PCL phase was significantly improved with the synergistic effect of P[MPEGMA-IL] and Gra. ${ }^{[34]}$ The morphological evolution of different systems is further schematically illustrated in Fig. 3.

\subsection{Thermal behavior of PLA/PCL/Gra/P[MPEGMA-IL] composites}

Fig. 4 exhibits the heat flow of PLA/PCL/Gra composites. As shown in Fig. 4a, the crystallization peak of PCL appeared near $25.39^{\circ} \mathrm{C}$ in PLA/PCL blend. However, for the blends containing Gra, the crystallization of PCL was fractionated into two peaks. In the PLA/PCL/0.25Gra composites, the first crystallization exotherm was recorded at $31.90{ }^{\circ} \mathrm{C}$ and the second crystallization exotherm occurred at a low temperature of $23.90{ }^{\circ} \mathrm{C}$. The former might be attributed to the nucleation effect of Gra, but the introduction of low content of Gra led to a decrease in the particle size of
PCL phase. Because the confined crystallization occurred and the crystallization capacity of PCL was reduced, the latter peak was lower than $25.39^{\circ} \mathrm{C}$ of PCL in the PLA/PCL system. With the addition of high amount of Gra, the crystallization peak of PCL shifted to an even lower temperature although the particle size of PCL phase was enlarged. Excessive Gra would hinder the movement of PCL chains, and then the crystallization process was delayed. On the other hand, the crystallinity of PLA was decreased as indicated in Table 2. There was no significant change in the cold crystallization temperatures of PLA/PCL and PLA/PCL/Gra system in Fig. 4b. Although Gra could serve as nucleation sites for PLA, it might also hinder the movement of PLA chains. Thus the crystallization ability of PLA has not changed much. The heat flow of PLA/PCL/P[MPEGMA-IL]/Gra composites was also investigated (Fig. 4c and 4d).

In Table 2, the thermal parameters obtained from DSC thermograms are shown, namely, cold crystallization temperature $\left(T_{\mathrm{cc}}\right)$, crystallization temperature $\left(T_{\mathrm{c}}\right)$, melting temperature $\left(T_{\mathrm{m}}\right)$ and crystallization degree $\left(X_{\mathrm{c}}\right)$ related to PLA and PCL. According to Fig. $4 \mathrm{c}$ and Table 2, the $T_{\mathrm{c}}$ and $X_{\mathrm{c}}$ of PCL decreased after adding 4P[MPEGMA-IL] because the decrease of PCL phase size would lead to the confined crystallization and reduce the crystallization capacity of PCL. ${ }^{[35]}$ But the $X_{\mathrm{c}}$ of PCL was increased after adding 
Table 2 Thermal properties of PLA/PCL blends filled with Gra and P[MPEGMA-IL].

\begin{tabular}{|c|c|c|c|c|c|c|c|c|c|c|}
\hline \multirow[b]{2}{*}{ Sample } & \multicolumn{5}{|c|}{ PLA } & \multicolumn{5}{|c|}{ PCL } \\
\hline & $\begin{array}{c}T_{c c} \\
\left({ }^{\circ} \mathrm{C}\right)\end{array}$ & $\begin{array}{l}\Delta H_{c c} \\
\left(\mathrm{Jg}^{-1}\right)\end{array}$ & $\begin{array}{c}T_{m} \\
\left({ }^{\circ} \mathrm{C}\right)\end{array}$ & $\begin{array}{l}\Delta H_{\mathrm{m}} \\
\left(\mathrm{Jg}^{-1}\right)\end{array}$ & $\begin{array}{l}X_{c} \\
(\%)\end{array}$ & $\begin{array}{c}T_{c} \\
\left({ }^{\circ} \mathrm{C}\right)\end{array}$ & $\begin{array}{l}\Delta H_{c} \\
\left(\mathrm{Jg}^{-1}\right)\end{array}$ & $\begin{array}{c}T_{m} \\
\left({ }^{\circ} \mathrm{C}\right)\end{array}$ & $\begin{array}{l}\Delta H_{m} \\
\left(\mathrm{Jg}^{-1}\right)\end{array}$ & $\begin{array}{c}X_{c} \\
(\%)\end{array}$ \\
\hline PLA/PCL & 106.6 & 19.2 & 166.9 & 23.3 & 6.3 & 25.4 & 15.4 & 54.4 & 10.6 & 22.5 \\
\hline PLA/PCL/0.25Gra & 108.6 & 19.4 & 167.2 & 22.4 & 4.5 & $23.9 / 31.9$ & 15.6 & 54.8 & 12.2 & 25.9 \\
\hline PLA/PCL/0.50Gra & 105.7 & 19.2 & 166.6 & 23.0 & 5.8 & $14.4 / 33.2$ & 18.4 & 55.1 & 14.5 & 30.7 \\
\hline PLA/PCL/0.75Gra & 105.4 & 21.5 & 166.7 & 25.7 & 6.5 & $2.8 / 33.7$ & $4.7 / 8.2$ & 55.1 & 13.9 & 29.5 \\
\hline $\begin{array}{l}\text { PLA/PCL/ } \\
\text { 4P[MPEGMA-IL] }\end{array}$ & 104.2 & 22.3 & 167.1 & 26.7 & 6.9 & $0.3 / 24.3$ & $0.7 / 7.1$ & 54.3 & 7.7 & 16.3 \\
\hline $\begin{array}{l}\text { PLA/PCL/ } \\
\text { 8P[MPEGMA-IL] }\end{array}$ & 101.3 & 20.6 & 166.6 & 27.0 & 9.9 & $1.4 / 24.7$ & $0.5 / 8.7$ & 54.2 & 9.7 & 20.7 \\
\hline $\begin{array}{l}\text { PLA/PCL/0.25Gra/ } \\
\text { 4P[MPEGMA-IL] }\end{array}$ & 101.9 & 18.2 & 166.5 & 25.9 & 11.7 & $9.2 / 24.7$ & $0.6 / 12.5$ & 54.5 & 12.2 & 25.9 \\
\hline $\begin{array}{l}\text { PLA/PCL/0.25Gra/ } \\
\text { 8P[MPEGMA-IL] }\end{array}$ & 101.1 & 17.6 & 166.7 & 24.9 & 11.1 & $8.2 / 23.1$ & $1.7 / 10.1$ & 54.5 & 12.0 & 25.5 \\
\hline
\end{tabular}

8P[MPEGMA-IL] because the PCL phase size was increased. The $X_{\mathrm{c}}$ of PLA was improved owing to the incorporated P[MPEGMA-IL]. P[MPEGMA-IL] dispersed in the PCL phase, which formed ion clusters and performed heterogeneous nucleation for PLA. Besides, the plasticization effect of ionic moiety in P[MPEGMA-IL] could enhance the mobility of PLA chain. Hence, the addition of P[MPEGMAIL] reduced the $T_{c c}$ of PLA and led to an increase in the crystallization degree. ${ }^{[36]}$ Interestingly, the crystallization ability of PLA and PCL was simultaneously improved when the combined action of P[MPEGMA-IL] and Gra was analyzed, especially the $X_{\mathrm{c}}$ of PLA was significantly increased. P[MPEGMA-IL] could improve the dispersion of Gra, resulting in increased nucleating sites in the PLA matrix, and facilitate the movement of PLA chain segments because the combination of P[MPEGMA-IL] and Gra could provide nucleation sites for PCL. ${ }^{[34]}$ So the Gra and P[MPEGMA-IL] had a synergistic effect on the crystallization behavior of PLA and PCL.

\subsection{Dielectric behavior of PLA/PCL/Gra/P[MPEGMA-IL] composites}

Fig. 5a and $5 \mathrm{~b}$ shows the dielectric permittivity $\left(\varepsilon^{\prime}\right)$ and dielectric loss $\left(\varepsilon^{\prime \prime}\right)$ of PLA/PCL/Gra composites. The dielectric permittivity of the composites decreases with increasing frequency, which is caused by the dielectric relaxation of the dielectric material. Compared to PLA/PCL blends, the dielectric permittivity of the composites increases significantly with the addition of Gra. The degree of dispersion of Gra in the PLA/PCL matrix was close to the "percolation threshold" when the Gra was increased. ${ }^{[37]}$ But for PLA/PCL/0.50Gra and PLA/PCL/0.75Gra composites, the high content of Gra caused agglomeration, which was not conducive to its dispersion, resulting in no significant change in the dielectric permittivity of the blend. Fig. $5 b$ exhibits the dielectric loss of composites with frequency. When the Gra content was higher than the percolation threshold, a large number of conductive paths would cause leakage currents, which significantly increased the dielectric loss of the material at low frequencies.

The dielectric permittivity and dielectric loss of PLA/PCL/ P[MPEGMA-IL]/Gra at room temperature was also investigated (Fig. 5c and 5d). The dielectric permittivity of the PLA/PCL/P[MPEGMA-IL] blends was significantly higher than the PLA/PCL blends. During the polarization process, P[MPEGMA-IL] increased the polarization of the ion clusters and the interfacial polarization between PLA and PCL phase containing P[MPEGMA-IL]. The plasticization of the ionic liquid copolymer promoted the movement of the segments and improved the interfacial and orientation polarization of the blends. ${ }^{[38]}$ In addition, PCL dispersed into more phases in the PLA matrix after the addition of P[MPEGMA-IL] from the SEM images, which also enhanced the interfacial polarization and the dielectric permittivity. When adding P[MPEGMA-IL] modified Gra, the addition of P[MPEGMA-IL] promoted the better dispersion of Gra and facilitated the formation of interfacial area between polymer phase and modified Gra phase. The PLA/PCL/0.25Gra/8P[MPEGMA-IL] composite had the highest dielectric permittivity. Fig. 5b shows the relationship between the dielectric loss and the different frequency. The dielectric loss of the composites increased with increasing the content of Gra and P[MPEGMA-IL]. Because the presence of Gra and P[MPEGMA-IL] had large conductance losses and interfacial polarization at low frequency bands, and therefore its dielectric loss would be greater. The effect of modified Gra on the charge carrier motion mechanism was owing to the increase of the mobile charge carriers at the interface and the dielectric strength of the interfacial polarization.

3.4 Effect of Gra on morphology and dielectric properties of PLA/PCL/Gra/P[MPEGMA-IL] composites

Fig. 6 shows the DSC curves of the PLA/PCL/Gra/P[MPEGMA-IL] composites. The composites 

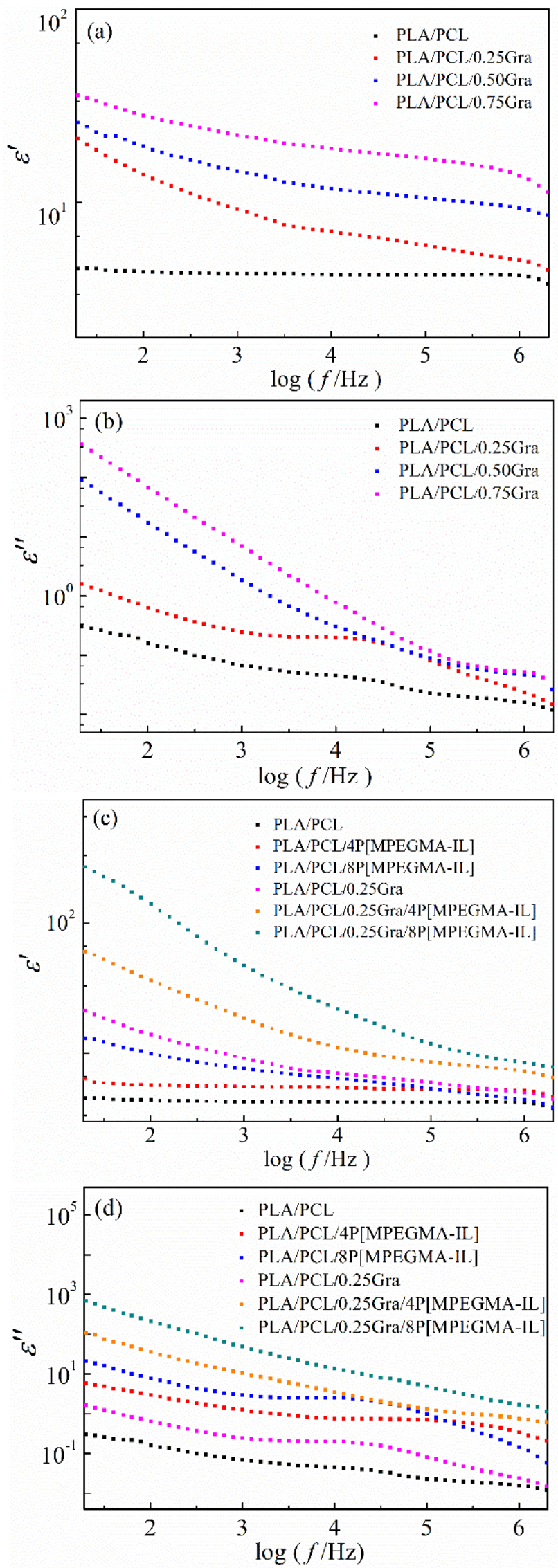

Fig. 5 Frequency dependence of (a) dielectric permittivity and (b) dielectric loss of PLA/PCL blends filled with Gra; (c) dielectric permittivity and (d) dielectric loss of PLA/PCL blends filled with Gra and P[MPEGMA-IL].
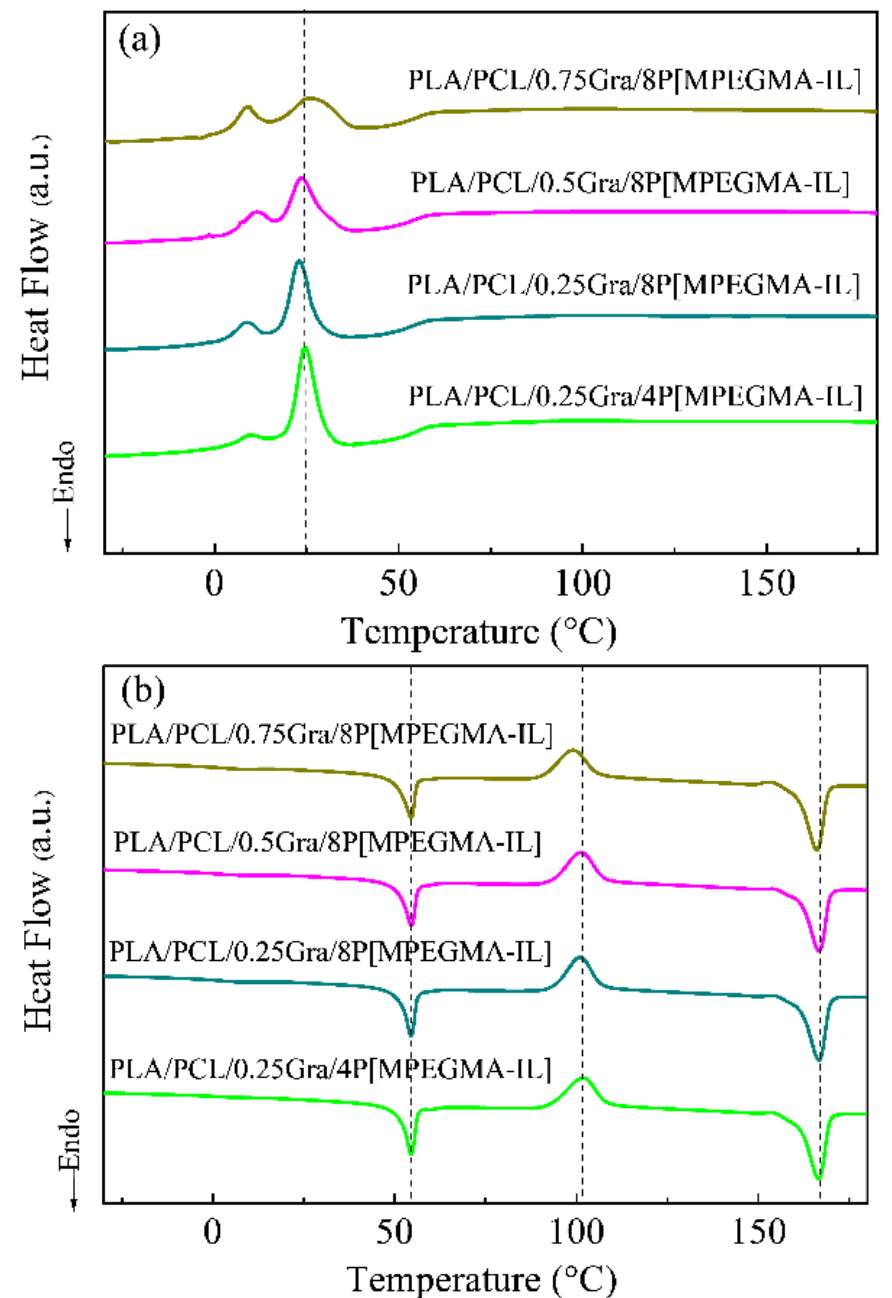

Fig. 6 DSC curves of PLA/PCL/Gra/P[MPEGMA-IL] composites: (a) cooling curves and (b) the second heating curve.

had a crystallization peak during the cooling process, which was the crystallization peak of PCL. No crystallization occurred during the cooling process due to the slow crystallization rate of PLA. PCL had obvious double crystallization peaks, and the enthalpy of crystallization gradually decreased with the increase of Gra content. This showed that the crystallization environment of PCL was in a chain space hindered state, and the crystallization performance was suppressed after adding high content of Gra. ${ }^{[39]}$ Fig. 6b shows the second heating curve of the composites. The melting enthalpy of PCL gradually decreased with increasing the Gra content, and the cold crystallization peak of PLA gradually was decreased. This indicated that the chain movement ability of PLA was increased. Fig. 7 shows the microstructure of the PLA/PCL/Gra/P[MPEGMA-IL] composites after etching with glacial acetic acid. It could be seen from the figure that the brittle cross-section showed scattered voids and presented a "sea-island" structure after etching the PCL phase. The dispersed phase PCL was still dispersed in the PLA phase in the form of spherical droplets, but the phase size was reduced with the increase of Gra content. ${ }^{[40]}$

After adding Gra, Gra hindered the movement of the PCL segment, and reduced the arrangement and aggregation of the 
PCL segment during the solution blending process. According to the DSC data, the size of the PCL phase decreased, the degree of hindered chain space was increased when adding Gra, and the PCL crystallization peak was broadened. This was mainly due to the increase of Gra content. In the smaller PCL phase, the crystal environment was in a state of hindered chain space, and it was difficult for PCL molecular chain to arrange the chain segments in a smaller space. At the same time, the high content of Gra hindered the movement of the chain segment, and the phenomenon of restricted crystallization was serious. Therefore, crystallization occurred at the low temperature of the bulk crystallization temperature. In addition, Gra played the role of heterogeneous nucleation, which provided a nucleation site for the arrangement of PLA segments and improved the crystallization ability of PLA. ${ }^{[41]}$ The high content of Gra hindered the PLA crystallization, which formed many imperfect crystal forms. During cold crystallization, conformational adjustment and molecular chain rearrangement occurred.
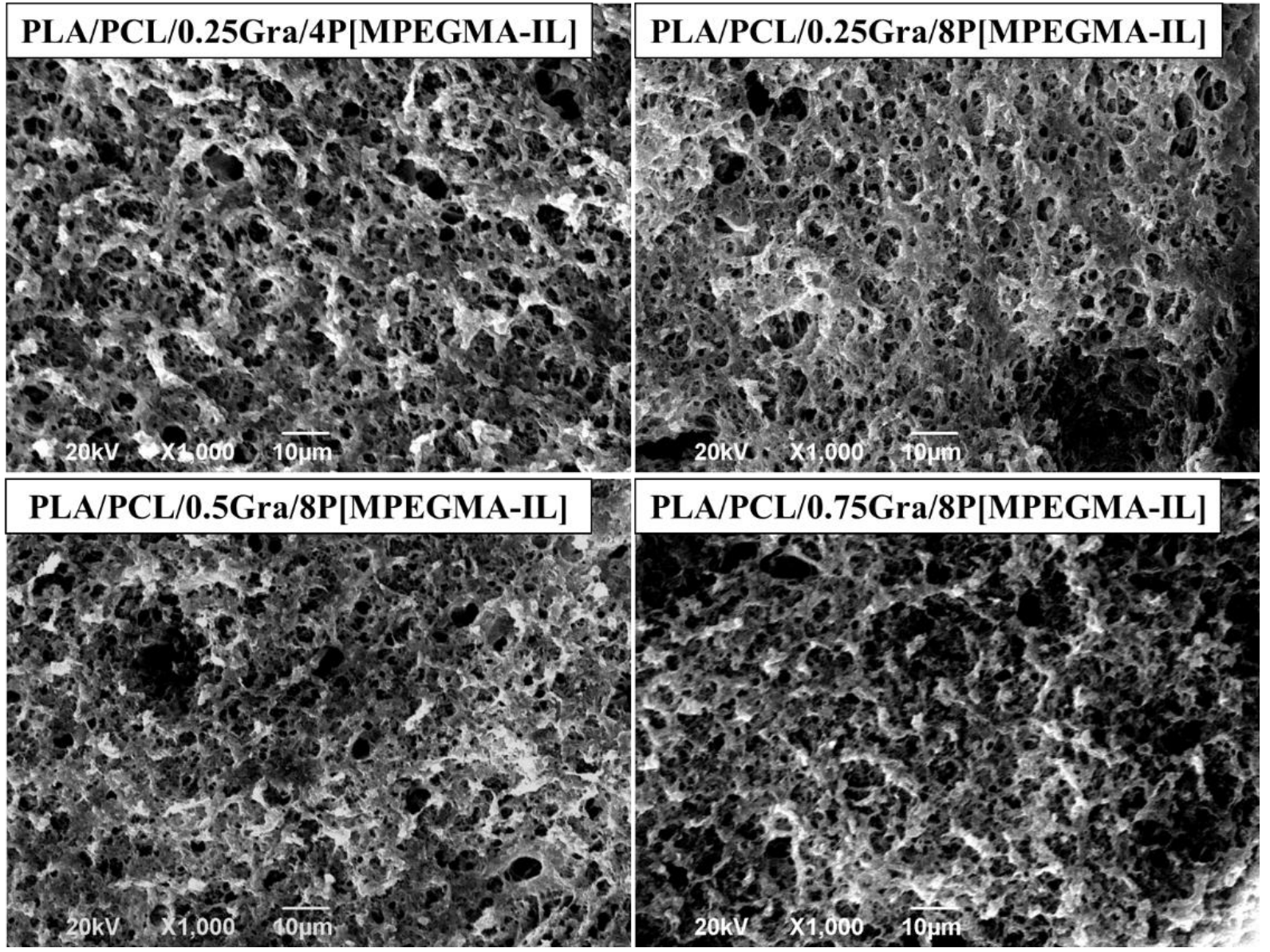

Fig. 7 SEM micrographs for the cryogenically fractured surfaces of (a) PLA/PCL/0.25Gra/4P[MPEGMA-IL], (b) PLA/PCL/0.25Gra/8P[MPEGMA-IL], (c) PLA/PCL/0.5Gra/8P[MPEGMA-IL] and (d) PLA/PCL/0.75Gra /8P[MPEGMA-IL].

Table 3 Thermal properties of PLA/PCL/Gra/P[MPEGMA-IL] composites.

\begin{tabular}{|c|c|c|c|c|c|c|c|c|c|c|}
\hline sample & \multicolumn{5}{|c|}{ PLA } & \multicolumn{5}{|c|}{ PCL } \\
\hline $\begin{array}{l}\text { PLA/PCL/0.25Gra/ } \\
\text { 4P[MPEGMA-IL] }\end{array}$ & 101.92 & 18.22 & 166.46 & 25.91 & 11.72 & $9.19 / 24.72$ & $0.61 / 12.45$ & 54.52 & 12.16 & 25.85 \\
\hline $\begin{array}{l}\text { PLA/PCL/0.25Gra/ } \\
\text { 8P[MPEGMA-IL] }\end{array}$ & 101.11 & 17.64 & 166.66 & 24.93 & 11.11 & $8.21 / 23.11$ & $1.66 / 10.05$ & 54.46 & 12.01 & 25.53 \\
\hline $\begin{array}{l}\text { PLA/PCL/0.75Gra/ } \\
\text { 8P[MPEGMA-IL] }\end{array}$ & 99.23 & 16.61 & 166.08 & 25.86 & 14.10 & $8.99 / 26.25$ & $2.45 / 6.62$ & 54.50 & 10.93 & 23.23 \\
\hline
\end{tabular}



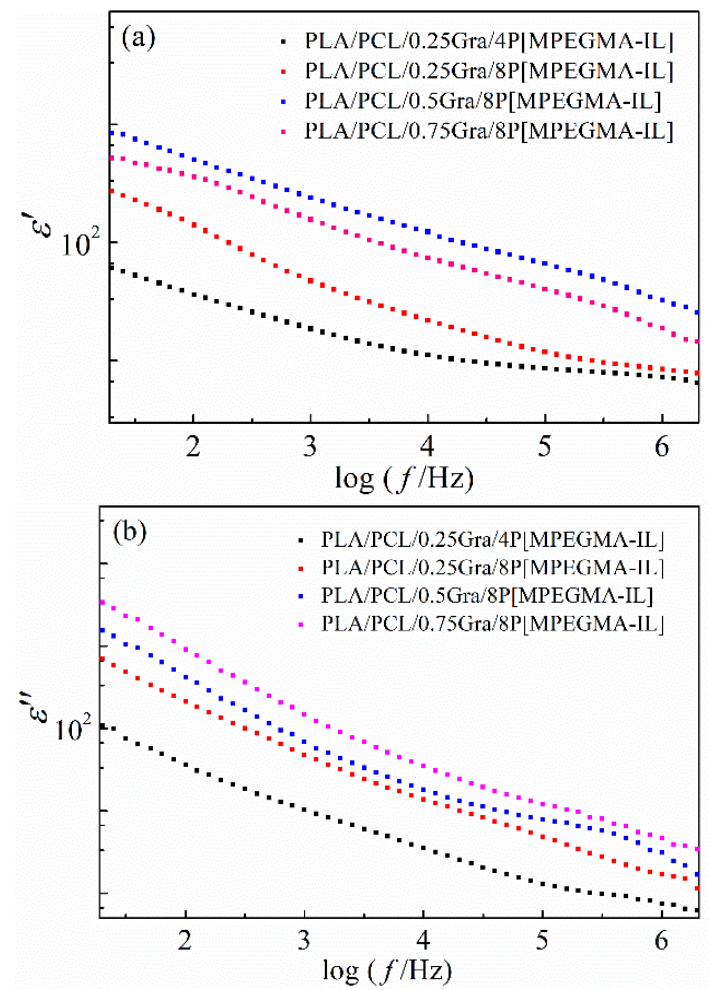

Fig. 8 Frequency dependence of dielectric permittivity and dielectric loss for PLA/PCL/Gra/P[MPEGMA-IL] composites.

Fig. 8 shows the dielectric properties of PLA/PCL/Gra/ P[MPEGMA-IL] composites. It could be seen that the dielectric permittivity of the composites decreased with increasing frequency. Interfacial polarization and orientation polarization exist in the composites under the action of electric field. The interface polarization had sufficient response time to frequency, the composites exhibited a strong dielectric response at low frequencies. The dielectric permittivity of the composites was improved with increasing the Gra content. The space charge carriers were aggregated at the interface between Gra and the polymer, and thus tended to form interfacial polarization when Gra was dispersed in the composites. ${ }^{[42]}$ The interface with PLA and filler was increased due to the decreased dispersed phase of PCL, resulting in an enhanced interface polarization.

In addition, due to the formation of $\pi-\pi$ conjugation between P[MPEGMA-IL] and Gra, it promoted the dispersion of Gra and improved the dielectric properties of the composites. The dielectric properties of the composites were reduced after the addition of 0.75 wt $\%$ Gra, which reveals that adding high content Gra might make it easier to form a conductive path and a leakage current in the system, resulting in the reduction of dielectric properties of the composites. Fig. 8b shows the dielectric loss of the composites. The dielectric loss of the composites was decreased with increasing frequency. The large conductance loss and interface polarization appeared in the low frequency due to the presence of Gra. Gra had a high conductivity, and the conductive path formed by it would cause the leakage current, which would increase the dielectric loss of the composites at low frequencies.

\section{Conclusion}

PLA/PCL/P[MPEGMA-IL]/Gra blends were prepared by a solution blending and hot pressing. The crystallization of PLA and PCL was promoted by the combination use of P[MPEGMA-IL] and Gra because of the heterogeneous nucleation of Gra and ion cluster of P[MPEGMA-IL]. Furthermore, P[MPEGMA-IL] modified Gra could be mainly localized in the PCL domains, and the dispersion of PCL in the PLA matrix was improved. The good conductivity of Gra and the better dispersion of PCL in the blends made the PLA/PCL blends had a higher dielectric permittivity. The effect of modified Gra on the charge carrier motion mechanism was owing to the increase of the mobile charge carriers at the interfaces.

\section{Acknowledgments}

This research was supported by the National Natural Science Foundation of China (No. 51903002), Industrial Guidance Fund Project of Bengbu City and Hefei University of Technology (JZ2020YDZJ0334), Natural Science Foundation of Anhui Education Department (No. KJ2019A0774), Major science and technology projects of Anhui Province (201903a05020027), Anhui Jianzhu University PhD Startup Fund (2019QDZ22) and University Collaborative Innovation Project of Anhui province (GXXT2019-017), Hefei Key Technology Major R \&D Projects(No. J2019G19).

\section{Supporting information}

Not applicable

\section{Conflict of interest}

There are no conflicts to declare.

\section{References}

[1] Y. M. Lin, A. R. Boccaccini, J. M. Polak, A. E. Bishop and V. J. Maquet, Biomater. Appl., 2006, 21, 109-118, doi: $10.1177 / 0885328206057952$.

[2] N. J. Wu and H. Zhang, Mater. Lett., 2017, 192, 17-20, doi: 10.1016/j.matlet.2017.01.063.

[3] L. T. Lim, R. Auras and M. Rubino, Prog. Polym. Sci., 2008, 33, 820-852, doi: 10.1016/j.progpolymsci.2008.05.004.

[4] R. M. Rasal and D. E. Hirt, J. Biomed. Mater. Res. A., 2009, 88, 1079-1086, doi: 10.1002/jbm.a.32009.

[5] M. Murariu, L. Bonnaud, P. Yoann, G. Fontaine, S. Bourbigot and P. Dubois, Polym. Degrad. Stab., 2010, 95, 374381, doi: 10.1016/j.polymdegradstab.2009.11.032.

[6] H. Bai, H. Xiu, J. Gao, H. Deng, Q. Zhang, M. Yang and Q. Fu, ACS Appl. Mater. Inter., 2012, 4, 897-905, doi: 10.1021/am201564f.

[7] B. U. Nam, K. D. Min and Y. Son, Mater. Lett., 2015, 150, 118-121, doi: 10.1016/j.matlet.2015.03.019.

[8] J.M. Zhang, S.W. Wang, Y. H. Qiao and Q. Li, Colloid. Polym. Sci., 2016, 294, 1779-1787 doi: 10.1007/s00396-0163940-5. 
[9] A. Rigoussen, P. Verge, J. M. Raquez, Y. Habibi and P. Dubois, Eur. Polym. J., 2017, 93, 272-283. doi: 10.1016/j.eurpolymj.2017.06.004

[10] G. Kfoury, J. M. Raquez, F. Hassouna, J. Odent, V. Toniazzo, D. Ruch and P. Dubois, Front. Chem., 2013, 1, 32. doi: 10.3389/fchem.2013.00032

[11] S. Rathi, X. Chen, E.B. Coughlin, S. L. Hsu, C. S. Golub and M. J. Tzivanis, Polymer, 2011, 52, 4184-4188 doi: 10.1016/j.polymer.2011.07.032.

[12] H.W. Bai, C. M. Huang, H. Xiu and Y. Gao, Polymer, 2013, 54, 5257-5266. doi: 10.1016/j.polymer.2013.07.051.

[13] I. Fortelny, A. Ujčić, L. Fambri and M. Slouf, Front. Mater., 2019, 6, 206, doi: 10.3389/fmats.2019.00206.

[14] X. Xiao, V. S. Chevali, P. Song, B. Yu, Y. Yang and H. Wang, Compos. Commun., 2020, 100385, doi: 10.1016/j.coco.2020.100385.

[15] P. Wang, D. Zhang, Y. Zhou, Y. Li, H. Fang, H. Wei and Y. Ding, Ionics, 2018, 24, 787-795, doi: 10.1007/s11581-0172234-3.

[16] A. Chuvilin, E. Bichoutskaia, M. C. Gimenez-Lopez, T. W. Chamberlain, G. A. Rance, N. Kuganathan, J. Biskupek, U. Kaiser and A. N. Khlobystov, Nat. Mater., 2011, 10, 687-692, doi: $10.1038 /$ nmat3082.

[17] T. Takayama and M. Todo, J. Mater. Sci., 2010, 45, 62666270, doi: $10.1007 / \mathrm{s} 10853-010-4822-8$.

[18] J. Hou, Z. Liu and P. Zhang, J. Power. Sources, 2013, 224, 139-144, doi: 10.1016/j.jpowsour.2012.09.091.

[19] S. Stankovich, D. A. Dikin, G. H. Dommett, K. M. Kohlhaas, E. J. Zimney, E. A. Stach, R. D. Piner, S. T. Nguyen and R. S. Ruoff, Nature, 2006, 442, 282-286. doi: 10.1038/nature04969.

[20] L.S. Schadler, S. C. Giannaris and P. M. Ajayan, Appl. Phys. Lett., 1998, 73, 3842-3844, doi: 10.1063/1.122911.

[21] C. G. Lee, X.D. Wei, J.W. Kysar and J. Hone, Science, 2008, 321, 385-388, doi: 10.1126/science.1157996.

[22] D. Bhadra, J. Sannigrahi, B. K. Chaudhuri and H. Sakata, Polym. Compos., 2012, 33, 436-442, doi: 10.1002/pc.22136.

[23] J. S. Wilkes, Green Chem., 2002, 4, 73-80, doi: 10.1039/B110838G.

[24] M. Armand, F. Endres, D. R. Macfarlane, H. Ohno and B. Scrosati, Nat. Mater., 2009, 8, 621-629, doi: 10.1038/nmat2448. [25] B. S. Bouakaz, A. Habi, Y. Grohens and I. Pillin, Appl. Clay Sci., 2017, 139, 81-91, doi: 10.1016/j.clay.2017.01.014.

[26] N. V. Plechkova and K. R. Seddon, Chem. Soc. Rev., 2008 , 37, 123-150, doi: 10.1039/B006677J.

[27] S. Yaragalla, B. Sindam, J. Abraham1, K. J. Raju, N. Kalarikkal and S. Thomas, J. Polym. Res., 2015, 22, 137, doi: 10.1007/s10965-015-0810-7.

[28] E. Laredo, M. Grimau, A. Bello, D. F. Wu, Y. S. Zhang and D. P. Lin, Biomacromolecules, 2010, 11, 1339-1347, doi: 10.1021/bm100135n.

[29] J. Abraham, M. P. Arif, P. Xavier, S. Bose, S. C. George, N. Kalarikkal and S. Thomas, Polymer, 2017, 112, 102-115, doi: 10.1016/j.polymer.2017.01.078.

[30] H. H. Le, X. T. Hoang, A. Das, U. Gohs, K. W. Stoeckelhuber, R. Boldt, Carbon, 2012, 12, 4543-4556 doi: 10.1016/j.carbon.2012.05.039.

[31] D. F. Wu, D. P. Lin, J. Zhang, W. Zhou, M. Zhang, Y. Zhang, D. Wang and B. Lin, Macromol. Chem. Phys. 2011, 212, 613-626, doi: 10.1002/macp.201000579.
[32] P. Wang, P. Xu, H. B. Wei, H. G. Fang and Y. S. Ding, J. Appl. Polym. Sci., 2018, 135, 46161, doi: 10.1002/app.46161.

[33] F. Laoutid, D. François, Y. Paint, L. Bonnaud and P. Dubois, Macromol. Mater. Eng., 2013, 298, 328-338, doi: 10.1002/mame.201200047.

[34] P. Xu, X. Luo, Y. Zhou, Y. Yang and Y. Ding, Thermochim. Acta, 2017, 657, 156-162, doi: 10.1016/j.tca.2017.10.005.

[35] S. Bose, A. R. Bhattacharyya, P. V. Kodgire and A. Misra, Polymer, 2007, 48, 356-362, doi: 10.1016/j.polymer.2006.11.019.

[36] C. Yordanov and L. Minkova, Eur. polym. J., 2005, 41, 527-534, doi: 10.1016/j.eurpolymj.2004.10.034.

[37] S. Das and A. Ghosh, J. Phys. Chem. B, 2017, 121, 5422, doi: 10.1021/acs.jpcb.7b02277.

[38] M. P. Scott, C. S. Brazel, M. G. Benton, J. W. Mays, J. D. Holbrey, and R. D. Rogers, Chem. Commun., 2002, 13, 1370, doi: 10.1039/B204316P

[39] M. Weng and Z. Qiu, Macromolecules, 2014, 47, 83518358, doi: 10.1021/ma502019x.

[40] P. Xu, Z. Cui, G. Ruan and Y. Ding, Chinese J. Polym. Sci., 2019, 37, 243-8252, doi: 10.1007/s10118-019-2192-5.

[41] Y. Y. Liang, S. Yang, X. Jiang, G. J. Zhong, J. Z. Xu and Z. M. Li, J. Phys. Chem. B, 2015, 119, 4777-4787, doi: 10.1021/jp511742b.

[42] F. A. He, S.T. Lau, H.L.W. Chan and J.T. Fan, Adv. Mater., 2009, 21, 710-715, doi: 10.1002/adma.200801758.

\section{Author information}

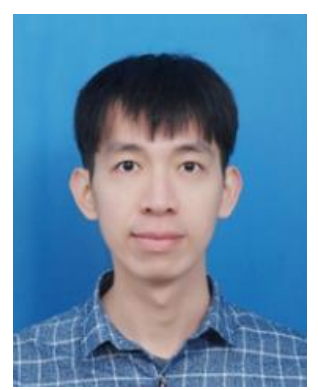

Yiyang Zhou is a PHD candidate in the School of Chemistry and Chemical Engineering at Hefei University of Technology. Dr Zhou's research interests focus on high performance polymer composites and the radiation effect on polymer materials.

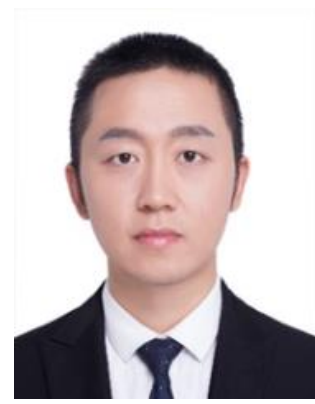

Ping Wang is a professor in the School of Materials and Chemical Engineering at Anhui Jianzhu University. Prof Wang's research interests mainly focus on the structure and properties of polymer composites and frontal polymerization.

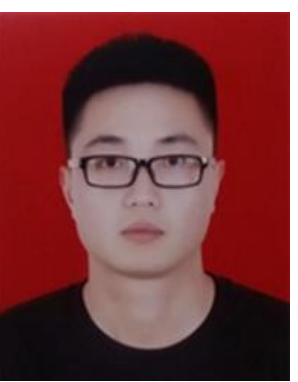

Gang Ruan is a master student graduated from the School of Chemistry and Chemical Engineering at Hefei University of Technology. Gang Ruan works on the fabrication and application of dielectric polymer composites. 


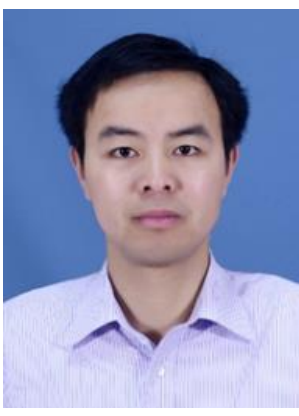

$P e i \mathrm{Xu}$ is an associate professor in the School of Chemistry and Chemical Engineering at Hefei University of Technology. Dr Xu's research interests focus on the dielectric and conductive properties of carbon nanofiller-based polymer composites.

Publisher's Note Engineered Science Publisher remains neutral with regard to jurisdictional claims in published maps and institutional affiliations.

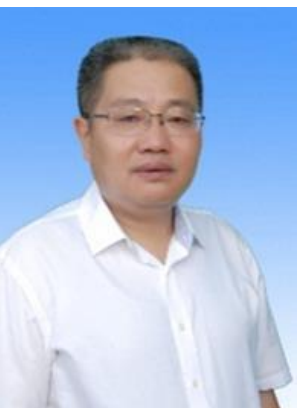

Yunsheng Ding is a professor in the School of Chemistry and Chemical Engineering at Hefei University of Technology. Prof Ding's research interests focus on the fabrication and application of high performance polymer composites. His research work can be applicable towards nuclear cables, marine anti-corrosion, high-speed rail and other industrial fields. 